\title{
Neurociências, artes gráficas e saúde pública: as novas advertências sanitárias para maços de cigarros
}

\author{
Neurosciences, graphic arts, and public health: new health \\ warnings on cigarette packaging
}

Billy E.M. Nascimento Laboratório de Neurobiologia II, IBCCF/URFJ

\author{
Av. Carlos Chagas Filho, 373 \\ Ed. do Centro de Ciências, bl.G/ \\ s.019 \\ 21941-902 - Rio de Janeiro - RJ - \\ Brasil \\ billy@biof.ufrj.br
}

Nilton Gamba Jr.

Departamento de Artes \& Design/ PUC-RJ

gambarj@terra.com.br

Leticia de Oliveira

Mirtes G. Pereira

Departamento de Fisiologia e

Farmacologia/Instituto Biomédico/ UFF

Idol@vm.uff.br

mirtes@vm.uff.br

\section{Rejane Spitz}

Departamento de Artes \& Design/ PUC-RJ

rejane@puc-rio.br

\section{Sonia Gleiser}

Laboratório de Neurofisiologia da Cognição/UFF

soniaurca@yahoo.com.br

\section{Cristina Perez}

CristianeVianna

Tania Cavalcante

Divisão de Controle do Tabagismo/ Conprev/Inca

cperez@inca.gov.br

cfvianna@inca.gov.br

taniac@inca.gov.br

Eliane Volchan

Laboratório de Neurobiologia II, IBCCF/UFRJ

evolchan@biof.ufrj.br
NASCIMENTO, Billy E.M. et al. Neurociências, artes gráficas e saúde pública: as novas advertências sanitárias para maços de cigarros. História, Ciências, Saúde - Manguinhos, Rio de Janeiro, v.17, supl.1, jul. 2010, p.243-252.

Resumo

A exposição a produtos derivados do tabaco é considerada a mais importante causa de morte evitável no mundo. Ações de controle do tabagismo envolvem uma gama de intervenções para ajudar pessoas a parar de fumar e prevenir que jovens não se tornem dependentes. Advertências sanitárias com imagens mostradas em embalagens de cigarro são uma das formas mais efetivas de informar acerca das consequências do tabagismo. Pesquisas em neurobiologia da emoção demonstram que estímulos visuais afetam atitudes e comportamentos; estímulos agradáveis promovem predisposições para aproximação e os aversivos, afastamento. Os apelos positivos que o marketing da indústria tabagista induz em suas embalagens devem ser neutralizados por advertências que mostrem os riscos de fumar, desconstruindo o apelo prazeroso e induzindo predisposições de afastamento em relação ao produto.

Palavras-chave: controle do tabagismo; neurobiologia da emoção; advertências sanitárias.

\section{Abstract}

Exposure to tobacco products is considered theleading cause of avoidable death in the world. Tobacco control initiatives encompass a gamut of ways of helping people to quit smoking and keeping young people from ever becoming addicted. Pictorial health warnings on cigarette packaging constitute one of the most effective means of conveying information about the consequences of smoking. Research on the neurobiology of emotions shows that visual stimuli affect attitudes and behavior: pleasant stimuli prompt a tendency to approach while unpleasant stimuli prompt a tendency to avoid. The positive appeals placed on packaging by the tobacco industry's marketing departments should be neutralized by warnings that indicate the risks of smoking, thereby deconstructing any pleasurable appeal and prompting a tendency to avoid the product.

Keywords: tobacco control; neurobiology of emotions; health warnings. 
Billy E. M. Nascimento et al.

$\mathrm{N}$ os últimos anos uma alarmante epidemia tem-se espalhado pelo globo. O tabagismo, denominação de um dos subtipos de transtorno mental e comportamental ao uso de substância psicoativa, tem levado alto número de vítimas à morte prematura ou incapacitação física. As estatísticas são assustadoras: só no Brasil, duzentas mil pessoas morrem anual mente em decorrência da exposição aos produtos do tabaco. No século XX o consumo de tabaco matou cem milhões de pessoas em todo o planeta. As previsões para o século XXI são ainda mais dramáticas. Até 2030 serão oito milhões de mortes a cada ano, 80\% nos países em desenvolvimento. Se nada for feito para a redução do consumo, um bilhão de pessoas morrerá neste século pelo uso direto ou indireto de tabaco.

As folhas da planta tabaco (Nicotiana tabacum), processadas, podem gerar inúmeros tipos de produtos. No Brasil, os produtos derivados do tabaco podem ser encontrados mais comumente na forma de cigarros, charutos e cachimbos (inalação), fumo de rolo (mastigação) e rapé (aspiração). Todos esses produtos, quando consumidos, contribuem para que o tabagismo constitua a principal causa de morte evitável e fator de risco em seis das oito doenças que mais matam no mundo, entre elas infarto do miocárdio, acidente vascular cerebral, doença pulmonar obstrutiva crônica e tuberculose (WHO, 2008).

A preocupação das autoridades de saúde em todo o mundo para conter tal epidemia tem feito com que diversos esforços convirjam para aumentar a cessação entre os fumantes e diminuir a iniciação entre os jovens. Evidência desse processo foi a formulação do primeiro tratado mundial no âmbito da saúde, negociado sob os auspícios da Organização Mundial da Saúde. A Convenção-Quadro Para Controle do Tabaco (WHO, 2005), como ficou conhecida, é hoje um dos tratados de maior adesão na história das Nações Unidas, tendo 164 países signatários. Em suas diretrizes, a Convenção-Quadro estabelece uma série de regulamentos estratégicos para conter a epidemia tabagista, como aumento de impostos sobre produtos, proteção à fumaça dos cigarros (fumo passivo), banimento de qualquer tipo de propaganda, políticas de prevenção para os jovens, apoio aos dependentes e esclarecimento acerca das consequências do uso do tabaco.

O Brasil teve papel ativo na formulação e implementação da Convenção-Quadro, tendo ratificado sua participação em 2005 . Tido como exemplo mundial na área de controle do tabagismo, nosso país tem desenvolvido longa e histórica luta para ampliar as políticas de saúde pública no sentido de diminuir o consumo de produtos derivados do tabaco. Ao longo desse período, consolidaram-se diversas ações de caráter educativo, legislativo, econômico e de atenção à saúde. Essas ações visam contribuir para prevenir a iniciação, promover a cessação e proteger a população dos riscos do tabagismo passivo (Brasil, 2009).

Entre as mais importantes ações de controle do tabagismo adotadas no Brasil nos últimos dez anos, destacam-se a proibição de patrocínio de eventos culturais e esportivos pelas companhias de tabaco e a restrição da exibição de propagandas em rádio, TV, jornais e outras mídias - só permitidas nos pontos internos de venda. Com seu alto apelo emocional, tais propagandas influenciaram diversas gerações, disseminando imagens que procuraram associar o cigarro a glamour, rebeldia, status, independência, espírito de aventura, beleza, sexualidade e conquistando, assim, grande número de jovens.

Com o fim da propaganda, o meio mais efetivo de a indústria estimular a venda e o consumo de cigarros passou a ser sua própria embalagem. Não importa para qual produto, 
embalagens desempenham papel principal nas estratégias de marketing das indústrias para atrair consumidores. Um determinado invólucro deve rapidamente atrair a atenção e gerar aproximação para que, então, o consumidor efetivamente compre o que ele contém. Isso não é diferente com as embalagens dos produtos de tabaco, especialmente de cigarros.

Interessa observar o que os documentos secretos da indústria do tabaco dizem sobre suas estratégias de marketing. Esse material foi disponibilizado livremente a partir de uma série de ações judiciais empreendidas pelo governo dos Estados Unidos contra as indústrias tabagistas. Foi determinada, então, a exposição dos documentos internos de diversas empresas, incluindo as que detêm o mercado de cigarros no Brasil. Essa documentação pode ser acessada a partir do sítio da Biblioteca Legal de Documentos do Tabaco, ligada à Universidade da Califórnia em São Francisco (University of Califórnia, 2007).

Os objetivos quanto ao desenvolvimento de características para atrair o público jovem são bem claros, como lemos nos documentos da R.J. Reynolds, fabricante da marca Camel:

Se a companhia quiser sobreviver e prosperar no longo prazo, devemos conseguir uma fatia de mercado jovem... Assim nós precisamos de novas marcas desen hadas para serem particularmente atraentes para o jovem fumante, e que ideal menteagradem, ao mesmo tempo, todos os fumantes... Talvez essas questões possam ser melhor abordadas consideran do os fatores que influenciam os pré-fumantes a experimentarem um cigarro, aprender a fumar, e setornar fumantes definitivos (Teague, 1973; tradução livre).

Os fabricantes têm a clara noção de que o primeiro contato dos adolescentes com o cigarro é uma experiência desagradável devido ao efeito aversivo da nicotina e ao sabor forte do produto. Visando diminuir essa aversão, trabal ham ativamente, através de simbolismos próprios da fase da adolescência, para motivá-los a experimentar e a usar o cigarro como um passaporte para o mundo adulto.

O primeiro cigarro é uma experiência ruim para o principiante. Para dar conta do fato de que o fumante iniciante tolerará as sen sações desagradáveis (do primeiro cigarro) nós precisamos evocar motivação psicossocial. Fumar um cigarro para o iniciante éum ato simbólico. O fumante está dizendo ao mundo: "Esse é o tipo de pessoa que sou". Certamente existem outras variações destetema: "Eu não sou mais criança da minha mãe", “Eu sou forte”, “Eu sou um aventureiro", "Eu não sou quadrado" ... . À medida que a força do simbolismo psicossocial diminui, o efeito farmacológico assoma para manter o hábito (Phillip M orris, 1969; tradução livre).

Com evidências tão marcantes sobre os objetivos da indústria tabagista, intervenções para alertar atuais ou possíveis usuários tornam-se extremamente necessárias. No Brasil, a regulamentação dos invólucros de produtos derivados do tabaco já havia determinado, desde 1989, a inclusão da frase "O Ministério da Saúde adverte: Fumar é prejudicial à saúde" na lateral das embalagens. Mas foi a partir de 2001 que as advertências sanitárias ganharam destaquee evidência. Por lei foi determinado queas mesmas ocupassem total mente uma das principais faces das embalagens e contivessem imagens ilustrativas dos males do tabagismo, além de exibir o número de telefone do Disque Saúde - Pare de Fumar.

Sendo o segundo país no mundo a adotar a estratégia de incluir advertências pictóricas nas embalagens dos produtos derivados do tabaco (o primeiro foi o Canadá), o Brasil conquistou grande avanço no controle do tabagismo, implementando precocemente um 
Billy E. M. Nascimento et al.

dos principais artigos da Convenção-Quadro. O artigo 11 determina que todos os produtos de origem do tabaco devem conter em suas embalagens advertências sobre os males causados por seu uso. Segundo o mesmo artigo, advertências devem ocupar $50 \%$ ou mais da área principal da embalagem e não devem ser inferiores a 30\%. Devem ser grandes, visíveis, legíveis e claras, podendo incluir fotografias ou pictogramas.

No Brasil, a introdução de dois grupos de advertências com imagens, nove entre 2001 e 2004 e dez entre 2004 e 2008, aliada a outras intervenções já relatadas, contribuiu para considerável redução do número de fumantes no país. Os resultados positivos são traduzidos na diminuição da proporção de fumantes na população acima de 18 anos: de 34,8\% em 1989 para 22,4\% em 2003 (Monteiro et al., jul. 2007, p.527-534) e 16\% em 2006 (Brasil, 2007). Essa prevalência está bem abaixo de países como Espanha (33,2\%), França (29,9\%), EUA $(23,2 \%)$ e Argentina (29,7\%) (WHO, 2008).

Quando a inclusão de advertências com fotos nas embalagens dos produtos de tabaco tornou-se obrigatória, algumas companhias passaram a promover a venda de cigarreiras de metal, capas para os maços e outros artefatos para induzir o fumante a cobrir as advertências. Também passaram a inserir pequenos panfletos com superfície autocolante e com propaganda da marca, no mesmo formato e tamanho das advertências sanitárias, para o mesmo fim. Todas essas tentativas de enfraquecimento do impacto das advertências foram combatidas através de regulamentações expedidas pela Agência Nacional de Vigilância Sanitária (Anvisa).

Por outro lado, a reação da população foi muito positiva. Uma pesquisa de opinião realizada em 2002 pelo serviço Pare de Fumar - Disque Saúde mostrou que, dos 89.305 entrevistados ( $80 \%$ deles, fumantes), 92\% aprovaram a implementação das advertências pictóricas, 79\% disseram que as advertências deveriam ser mais impactantes e 90\% tomaram conhecimento do serviço Pare de Fumar - Disque Saúde através dos maços de cigarros.

O instituto de pesquisa Datafolha também realizou pesquisa de opinião no mesmo ano, encontrando resultados muito semelhantes: $76 \%$ dos entrevistados apoiaram a obrigatoriedade das imagens. Desse total, houve maior apoio entre os não fumantes $(77 \%)$, em comparação ao grupo de fumantes (73\%). Em meio aos que tinham curso superior ou médio, o apoio atingiu $83 \%$. Grande parte dos fumantes entrevistados (67\%) afirmou que as advertências os tinham estimulado a parar de fumar. Dos que possuíam renda até cinco salários mínimos, $73 \%$ disseram ter sentido vontade de deixar de fumar ao ver os novos maços. Dos que se encontravam na faixa de 25 a 34 anos, 73\% afirmaram ter pensado em largar o cigarro. A pesquisa também encontrou alta taxa de concordância, entre os entrevistados, com relação ao papel de prevenção das advertências com imagens que, segundo $70 \%$ deles, são muito eficientes para evitar a iniciação.

A percepção dos fumantes quanto às advertências com imagens também foi verificada no Inquérito Domiciliar Sobre Comportamentos de Risco e Morbidade Referida de Agravos Não Transmissíveis (Brasil, 2003), real izado junto à população acima de 15 anos. Das imagens de advertência, as que mais motivam o abandono da prática de fumar são aquelas que ilustram situações mais dramáticas, como a da mulher entubada com câncer de pulmão (79\%) e a do bebê prematuro (78\%). Quanto às que menos motivam, apontaram-se as que retratam situações sociais, como a de um pai fumando perto do filho, relacionada ao exemplo 
como fator para a iniciação de jovens; a que ilustra a dependência de nicotina, na qual um rapaz acende um cigarro no outro para passar a ideia de que se trata de fumante em cadeia, ou seja, um grande dependente; e a que sugere mau hálito, na qual um rapaz com um cigarro conversa com uma moça que parece enojada.

Aliado às pesquisas de opinião, um estudo científico alicerçado na pesquisa acadêmica procurou avaliar o impacto emocional dessas advertências. $\mathrm{O}$ trabalho foi desenvolvido por pesquisadores dos Laboratórios de Neurobiologia II da Universidade Federal do Rio de Janeiro (UFRJ) e Neurofisiologia do Comportamento da Universidade Federal Fluminense (UFF), em parceria com o Instituto Nacional do Câncer (Inca). A investigação baseou-se em um grande número de pesquisas em psicofisiologia da emoção, que revelam a influência explícita e implícita dos estímulos visuais sobre atitudes e comportamentos.

A metodologia empregada foi calcada em trabal ho de pesquisadores da Universidade da Flórida (Gainesville, Estados Unidos). Esse grupo, com o objetivo de desenvolver um conjunto-padrão de estímulos visuais para ser utilizado em estudos da emoção, elaborou um catálogo contendo centenas de fotografias, denominado Sistema Internacional de Fotografias Afetivas (Iaps, na sigla em inglês) (Lang, Bradley, Cuthbert, 2005). O mesmo grupo, utilizando os conceitos teóricos do modelo das bases motivacionais da emoção, construiu uma escala psicométrica para avaliação das fotografias do Iaps. Essa escala, chamada Self-Assessment Manikin (SAM), permite que o participante da pesquisa avalie determinada fotografia em dois grandes eixos do espaço afetivo-motivacional: a valência hedônica (grau de prazer) e a ativação emocional (grau de intensidade).

Para real ização da pesquisa, 212 jovens universitários foram voluntários para a avaliação das imagens - dezenas de fotografias, grande parte retirada do catálogo laps, e também as imagens das advertências sanitárias. Todas as imagens eram projetadas individualmente numa tela grande por tempo fixo, e cada uma delas era julgada pelos voluntários através de paradigma experimental padronizado conforme a escala SAM. Os resultados mostraram que as imagens foram consideradas bastante desagradáveis, porém não muito intensas ou ativantes. Também foi verificado que as advertências com cenas de pessoas fumando foram consideradas mais agradáveis para os fumantes, em comparação com os não fumantes (Nascimento et al., 2008).

A conclusão dessa pesquisa apontou algumas estratégias para a elaboração de novas advertências. Para aumentar a eficiência, as imagens deveriam evitar cenas de fumo e ser mais assustadoras, ou seja, mais intensas. Imagens indicativas de risco de vida e/ou contendo lesões corporais são as que mais aumentam respostas ligadas ao comportamento de afastamento e repulsa. Uma vez que um dos objetivos das advertências em embalagens de produtos de tabaco está ligado à diminuição do apelo ao prazer e à promoção de repulsa ao produto, as advertências poderiam utilizar conteúdos que otimizassem o processamento cerebral a predisposições aversivas.

Tendo em vista que advertências gráficas devem ser substituídas regularmente, para que não percam o impacto, e visando ampliar a divulgação de informações sobre os mal efícios do tabagismo, no final de 2006 o Ministério da Saúde passou a investir esforços para inovar na construção de um novo conjunto de advertências sanitárias. Com esse objetivo, sob a liderança do Inca, foi criado um Grupo de Estudos formado pela Divisão de Controle do 
Billy E. M. Nascimento et al.

Tabagismo do Inca, a Gerência de Produtos Derivados do Tabaco da Agência Nacional de Vigilância Sanitária, os Laboratórios de Neurobiologia II da UFRJ e de Neurofisiologia do Comportamento da UFF, e o Departamento de Artes \& Design da Pontifícia Universidade Católica do Rio de Janeiro (Brasil, 2009).

Os Laboratórios da UFR e UFF forneceram embasamento teórico e experimental no campo da psicofisiologia da emoção, para a seleção de categorias de imagens mais adequadas às advertências e para os testes de sua eficácia, ao passo que o Departamento da PUC-Rio forneceu embasamento teórico e conhecimento técnico no campo do design, para a criação das imagens e a formulação de novo layout, melhorando a visualização e a leitura das advertências. O Inca e a Anvisa forneceram o suporte técnico para abordagem dos temas que seriam contemplados.

Diversos trabalhos têm demonstrado a eficácia da utilização de mensagens amedrontadoras. Além de promover repulsa, tais mensagens podem gerar associação negativa envolvendo a embalagem e, por conseguinte, seu produto. Os mecanismos de associação compõem um dos princípios básicos da teoria de aprendizado em psicologia. Imagens agradáveis que acompanham um objeto causarão associação com emoções positivas. Em contrapartida, uma imagem repulsiva promoverá forte associação entre o objeto e emoções negativas, reforçada pela quantidade de vezes que o fumante manipula o maço (se ele fuma 20 cigarros por dia, manipula o maço 7.300 vezes ao ano!), índice de exposição raramente alcançado em campanhas publicitárias.

A equipe de Design da PUC-Rio desenvolveu taxonomia baseada na área de estudos culturais através de pesquisa e classificação de similares, estabelecendo critérios para construção dos protótipos das novas advertências. Esses critérios geraram três grupos principais de imagens: as metafóricas (ex: risco de derrame), as realistas com abordagem científica (ex: gangrena) e as realistas com viés jornalístico (ex: parto prematuro). Tal metodologia de análise permitiu evidenciar várias categorias, que foram utilizadas em todo o processo, desde a avaliação quantitativa de cada grupo até questões de ordem ética e estética.

Com base no próprio briefing do projeto, foram elaborados outros critérios, tais como a atenção especial aos segmentos da população atualmente mais vulneráveis às estratégias de marketing das empresas de tabaco, como os jovens, as mulheres e as classes economicamente menos favorecidas, bem como a evitação de imagens que funcionassem como 'gatilhos', 'pistas' que podem despertar o desejo de fumar, como pessoas fuman do, cinzeiros, isqueiros e cigarros acesos.

Outras noções relevantes na elaboração dessas imagens vinculam-se à sintaxe visual e à fenomenologia da imagem, possibilitando critérios mais precisos para leitura mais clara do conceito de aversividade. Aspectos da análise da imagem como enquadramento, palheta de cores, contraste e nitidez foram preceitos fundamentais para ampliar o potencial de leitura dessas peças gráficas, tendo como foco o resultado final de aversão.

Para a produção das imagens de forma a satisfazer a esses critérios, vários recursos técnicos foram utilizados, tanto na produção (maquiagem, mock-ups, iluminação etc.), como na pós-produção (computação gráfica).

Os protótipos desenvolvidos foram avaliados por 362 jovens com idade entre 18 e 24 anos, divididos em três níveis educacionais - ensino fundamental, ensino médio e ensino superior - 
com número bal anceado dehomensemulheres. Metadeda amostra de cada gênero era composta de fumantes. Foi utilizada a metodologia descrita em parágrafos anteriores e empregada no estudo das advertências que já haviam circulado e que estavam em circulação nos maços de cigarros no momento do estudo. Dessa vez a escala SAM foi empregada para os participantes julgarem tanto os protótipos das novas advertências como dezenas de imagens do catálogo laps, que serviram como controle do experimento. As sessões experimentais foram realizadas em sala ampla com o máximo de trinta participantes em cada uma. Como no estudo anterior, projetavam-se individualmente as imagens numa tela grande com boa visibilidade, por tempo fixo, para serem avaliadas. As instruções recebidas pelos voluntários não faziam distinção entre as imagens apresentadas, de modo que os participantes não tivessem conhecimento de que estavam avaliando imagens prototípicas para as futuras advertências.

Os resultados indicam que os protótipos foram considerados altamente aversivos e, comparados com os do primeiro estudo, que avaliou as imagens utilizadas nas 19 advertências veiculadas nos maços de cigarros, classificados como mais desagradáveis e com maiores escores na dimensão de ativação emocional, alcançando a meta estabelecida no início do projeto (Volchan et al., no prelo; Nascimento et al., submetido). Através dessa metodologia foi também possível estabelecer o escalonamento das imagens com maior conteúdo aversivo, índice utilizado como um dos critérios na escolha do novo grupo de advertências.

O layout das advertências foi também modificado para melhorar o impacto visual e semântico dos temas abordados. Na parte superior da advertência colocou-se palavra ou frase de destaque que resume o conteúdo da imagem, que aparece logo abaixo, seguida da advertência do Ministério da Saúde, com informação mais didática e aprofundada. Essa prerrogativa permite agora dois níveis de leitura, um mais fácil e rápido e o outro mais complexo e informativo. Por fim, a logo do Disque Saúde - Pare de Fumar, com o número de telefone do serviço, além de mais ampliada nessa versão, foi destacada ao ser posta em fundo preto, diferente do modelo anterior, em que era inserida na imagem, muitas vezes com pouca visibilidade.

O Brasil tem-se destacado mundialmente nas políticas de controle do tabaco. A união de órgãos de saúde pública e centros acadêmicos de pesquisa a trabalhar na formulação das novas advertências sanitárias para maços de cigarros tem sido considerada inovação de alcance promissor. As possi bilidades decorrentes dessa e futuras interações poderão apresentar resultados cada vez mais eficientes para a melhoria da saúde pública do país.

Advertências sanitárias podem manter o fumante alerta quanto aos prejuízos que podem vir a afetá-lo. Contribuem também para desestimular os jovens que estejam experimentando a droga. Seu caráter assustador e forte é um meio pelo qual a mensagem pode ser incorporada de forma clara, explícita e implicitamente. Estampadas nos maços, as advertências podem interferir no apelo que a propaganda transmite por meio de bonitos designs do próprio maço.

Através de trabal ho conjunto e pioneiro envolvendo distintas áreas de pesquisa, foi possível desenvolver advertências de alta relevância emocional, com intuito de proteger a população brasileira das graves consequências do tabagismo. Mais uma vez, o Brasil se coloca na vanguarda nos trabalhos de saúde pública, tornando-se modelo de inovação ao agregar conhecimentos acadêmicos a um mecanismo concreto de alerta para hábitos saudáveis. 
Billy E. M. Nascimento et al.
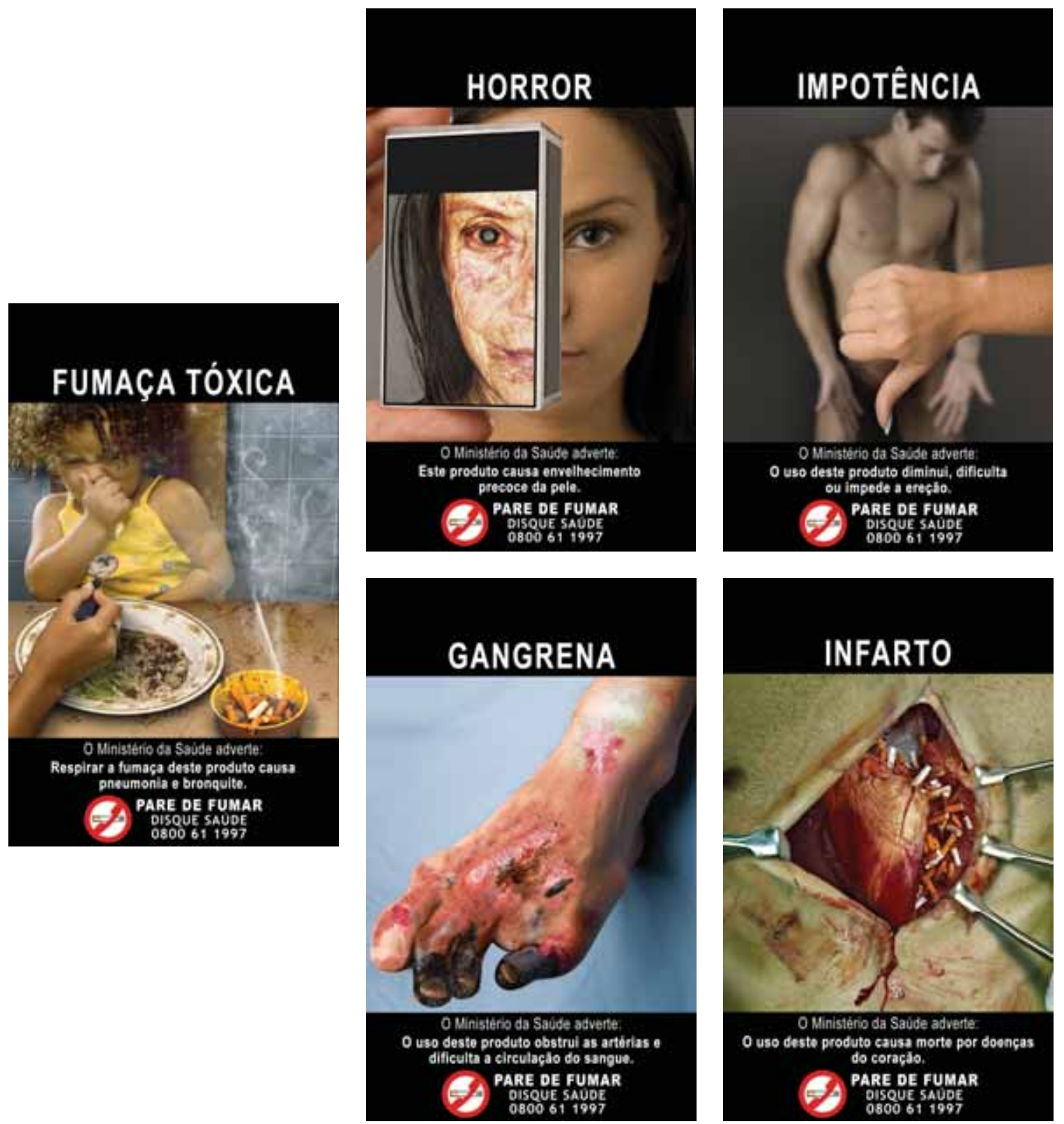

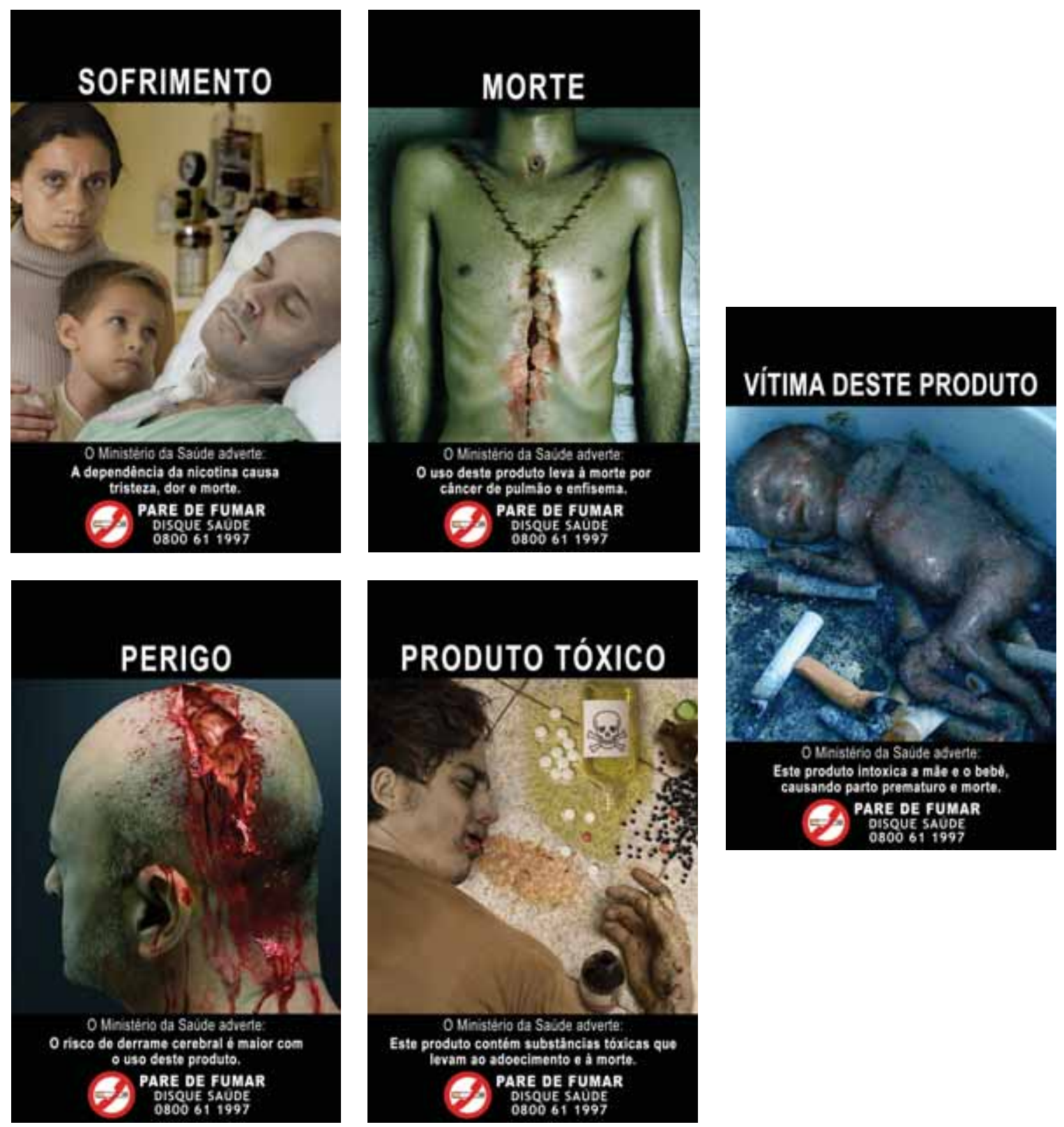
Billy E. M. Nascimento et al.

\section{REFERÊNCIAS}

BRASIL.

Ministério da Saúde. Instituto Nacional de Câncer. Coordenação de Prevenção e Vigilância. BRASIL: advertências sanitárias nas embalagens dos produtos de tabaco. Rio de Janeiro: INCA; CONPREV; Divisão de Controle do Tabagismo. 2009

BRASIL.

Ministério da Saúde. VIGITEL BRASIL 2006: vigilância de fatores de risco e proteção para doenças crônicas por inquérito telefônico. Disponível em: http://portal.saude.gov.br/ portal/arquivos/pdf/relatorio_vigitel_2006_ marco_2007.pdf. Acesso em: 15 maio 2009. 2007.

\section{BRASIL.}

Ministério da Saúde. Secretaria de Vigilância da Saúde. Inquérito domiciliar sobre comportamentos de risco e morbidade referida de agravos nãotransmissíveis. Rio de Janeiro: Instituto Nacional de Câncer. 2003.

LANG, Peter J.; BRADLEY, Margareth M.; CUTHBERT, Bruce N.

International affective picture system (IAPS): affective ratings of pictures and instruction manual - technical report a-6. Gainesville: University of Florida. 2005.

MONTEIRO, Carlos A. et al.

Population-based evidence of a strong decline in the prevalence of smokers in Brazil (1989-2003). Bulletin of the World Health Organization, New York, v.85, n.7, p.527-534. 2007.

NASCIMENTO, Billy E.M. et al. Avoidance of smoking: the impact of warning labels in Brazil. Tobacco Control, London, v.17, n.6, p.405-409. 2008.

NASCIMENTO, Billy E.M. et al.

Neuroscience and pictorial health warnings: a new integrated approach for tobacco control in Brazil. Bulletin of the World Health Organization, New York. submetido.

PHILLIP MORRIS.

Why on smokes. Disponível em: http:// www.pmdocs.com/PDF/1003287836_7848 0. PDF. 1969.

\section{TEAGUE, C.E.}

Research planning memorandum on some thoughts about new brands of cigarettes for the youth market. (RJ Reynolds). Disponível em: http:// legacy.library.ucsf.edu/tid/act68d00. 1973.

\section{UNIVERSITY OF CALIFÓRNIA.}

Legacy Tobaco Documents Library. San Francisco. Disponível em: http://legacy.library. ucsf.edu. Acesso em: 17 maio 2009. 2007.

VOLCHAN, Eliane et al.

Os males do fumo em destaque: o impacto emocional das imagens de advertência em maços de cigarros. Ciência Hoje, Rio de Janeiro. no prelo.

\section{WHO.}

World Health Organization. WHO report on the global tobacco epidemic, 2008: the mpower package. Geneva: WHO Press. 2008.

WHO.

World Health Organization. WHO framework convention on tobacco control. Geneva: WHO Press. 2005.

\section{uUuUUU}

